\title{
Corrigendum: Dental alloplastic bone substitutes currently available in Korea
}

\author{
Jeong-Kui Ku ${ }^{1,2}$, Inseok Hong ${ }^{3}$, Bu-Kyu Lee ${ }^{1}$, Pil-Young Yun ${ }^{4}$, Jeong Keun Lee ${ }^{5}$ \\ ${ }^{1}$ Department of Oral and Maxillofacial Surgery, Asan Medical Center, Seoul, \\ ${ }^{2}$ Department of Oral and Maxillofacial Surgery, Section of Dentistry, Armed Forces Capital Hospital, Seongnam, \\ ${ }^{3}$ Department of Oral and Maxillofacial Surgery, School of Dentistry and Institute of Oral Bioscience, \\ Research institute of Clinical Medicine of Chonbuk National University-Biomedical Research Institute of \\ Chonbuk National University Hospital, Chonbuk National University, Jeonju, \\ ${ }^{4}$ Department of Oral and Maxillofacial Surgery, Section of Dentistry, Seoul National University Bundang Hospital, Seongnam, \\ ${ }^{5}$ Department of Oral and Maxillofacial Surgery, Institute of Oral Health Science, Ajou University School of Medicine, Suwon, Korea
}

J Korean Assoc Oral Maxillofac Surg 2019;45:51-67.

https://doi.org/10.5125/jkaoms.2019.45.2.51

This correction is being published to correct the 4th author's affiliation in above article.

The authors apologize for this error.

Before correction

Jeong-Kui $\mathrm{Ku}^{1,2}$, Inseok Hong ${ }^{3}$, Bu-Kyu Lee ${ }^{1}$, Pil-Young Yun², Jeong Keun Lee ${ }^{4}$

${ }^{I}$ Department of Oral and Maxillofacial Surgery, Asan Medical Center, Seoul,

${ }^{2}$ Department of Oral and Maxillofacial Surgery, Section of Dentistry, Armed Forces Capital Hospital, Seongnam,

${ }^{3}$ Department of Oral and Maxillofacial Surgery, School of Dentistry and Institute of Oral Bioscience, Research Institute of Clinical Medicine of Chonbuk National University-Biomedical Research Institute of Chonbuk National University Hospital, Chonbuk National University, Jeonju,

${ }^{4}$ Department of Oral and Maxillofacial Surgery, Institute of Oral Health Science, Ajou University School of Medicine, Suwon, Korea

After correction

\author{
Jeong-Kui Ku ${ }^{1,2}$, Inseok Hong ${ }^{3}$, Bu-Kyu Lee ${ }^{1}$, Pil-Young Yun ${ }^{4}$ Jeong Keun Lee ${ }^{5}$ \\ ${ }^{\prime}$ Department of Oral and Maxillofacial Surgery, Asan Medical Center, Seoul, \\ ${ }^{2}$ Department of Oral and Maxillofacial Surgery, Section of Dentistry, Armed Forces Capital Hospital, Seongnam,
}

${ }^{3}$ Department of Oral and Maxillofacial Surgery, School of Dentistry and Institute of Oral Bioscience, Research institute of Clinical Medicine of Chonbuk National University-Biomedical Research Institute of Chonbuk National University Hospital, Chonbuk National University, Jeonju,

${ }^{4}$ Department of Oral and Maxillofacial Surgery, Section of Dentistry, Seoul National University Bundang Hospital, Seongnam,

${ }_{5}^{5}$ Department of Oral and Maxillofacial Surgery, Institute of Oral Health Science, Ajou University School of Medicine, Suwon, Korea

@ This is an open-access article distributed under the terms of the Creative Commons Attribution Non-Commercial License (http://creativecommons.org/licenses/by-nc/4.0/), which permits unrestricted non-commercial use, distribution, and reproduction in any medium, provided the original work is properly cited.

Copyright (C) 2019 The Korean Association of Oral and Maxillofacial Surgeons. All rights reserved. 